\title{
ARTERIAL ANOMALY AT THE OPTIC DISC*
}

BY

\section{ALEXANDER LINDSAY}

Manitoba Clinic, Winnipeg, Canada

THE following case displays a feature which, so far as I know, has not been previously described.

\section{Case Report}

A young girl first attended for eye examination on January 8, 1953, when she was 9 years of age. The visual acuity of each eye was $6 / 6$, there was no error of refraction, and the fundi were recorded as normal.

She was again examined on January 2,1958 , at the age of 14 years, when she was complaining of defective vision in the right eye. This eye had $2.25 \mathrm{D}$ sph. myopia, which accounted for the visual defect. The refraction of the left eye was still emmetropic. In the right fundus an artery pierced the lower part of the lamina cribrosa, crossed the centre of the optic disc, and joined the upper division of the central retinal artery behind its first bifurcation (Figure). No doubt this artery had been overlooked at the previous examination. With the slit lamp and Hruby lens, the contained blood column had a granular

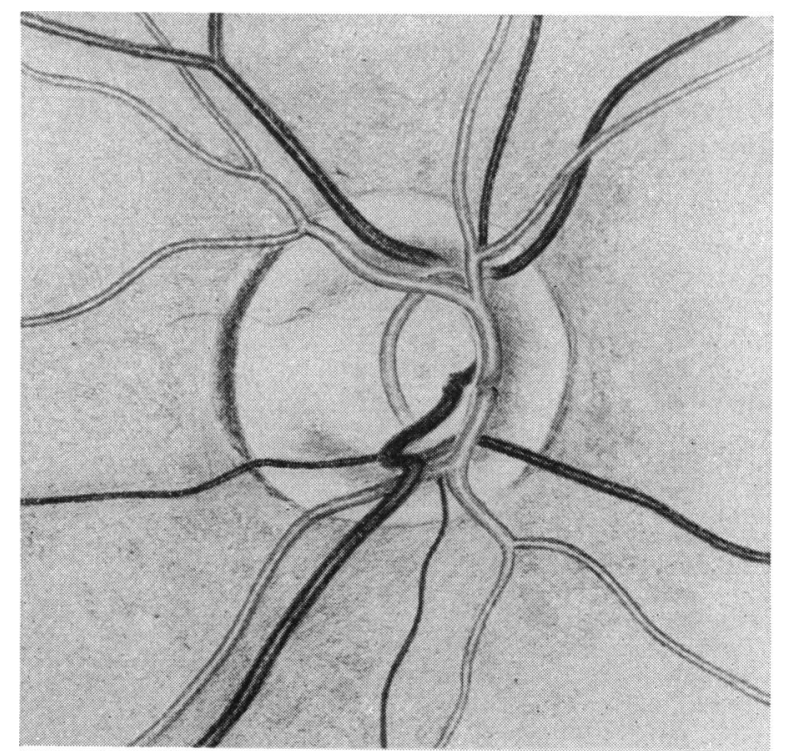

FIGURE.-Optic disc of the right eye showing arterial anomaly.

* Received for publication April 2, 1958 
appearance and moved slowly in an upward direction with a slight reverse movement between pulse beats. When pressure was applied to the eye the flow of blood ceased, and when the pressure was released there was a rapid reverse flow for 3 to 5 seconds before the original slow oscillating upward flow was resumed. None of these phenomena was present in the other visible intra-ocular blood vessels. The fundus of the left eye was normal.

The visual field of the right eye had an arcuate scotoma extending from the blind spot to the nasal meridian above fixation and between the 15 and 25 degree circles. This scotoma was detectable with a $1 \mathrm{~mm}$. but not with a $2 \mathrm{~mm}$. white object at a distance of one metre. The visual field of the left eye was within normal limits.

\section{Comment}

That the artery in question is not merely a branch of the upper division of the central retinal artery is evident from the direction of its blood flow. That it is not derived posteriorly from the central retinal artery is evident from the slow oscillating movement of its contained blood. It must therefore be derived from an artery in the orbit. The brief reversal of blood flow, which occurred on release of pressure applied to the eye, is probably a result of different hyperaemic responses in the orbit and retina. Pressure applied to the eye through the eyelid is transmitted to the orbit, and any reactive hyperaemia which might follow its release would be present in both locations. The hyperaemia with accompanying reduction of intra-vascular pressure would be more marked in the orbital vessels, since they, unlike the retinal vessels, are not exposed to the intra-ocular pressure.

The arcuate scotoma is not a result of poor circulation in the connecting artery, because the pressure in this artery is little different from that in the larger retinal arteries and any branches which it might give to the optic nerve-head would have an adequate circulation. The absence of a visual field defect in the left eye suggests that the arcuate scotoma and myopia in the right may have a common origin.

\section{Summary}

(1) An arterial anomaly at the optic disc is described.

(2) Reasons are given for considering it to be a connexion between arteries in the retina and orbit.

(3) An accompanying arcuate scotoma is considered to be unrelated to the arterial anomaly. 